\title{
Establishing the functional relevancy of white matter connections in the visual system and beyond
}

\author{
Mareike Grotheer $^{1,2}$ (D) $\cdot$ Emily Kubota ${ }^{3} \cdot$ Kalanit Grill-Spector $^{3,4}$
}

Received: 24 May 2021 / Accepted: 2 November 2021 / Published online: 30 November 2021

(C) The Author(s) 2021

\begin{abstract}
For over a century, researchers have examined the functional relevancy of white matter bundles. Consequently, many large-scale bundles spanning several centimeters have been associated in their entirety with specific brain functions, such as language or attention. However, these coarse structural-functional relationships are at odds with modern understanding of the fine-grained functional organization of human cortex, such as the mosaic of category-selective regions in ventral temporal cortex. Here, we review a multimodal approach that combines fMRI to define functional regions of interest within individual's brains with dMRI tractography to identify the white matter bundles of the same individual. Combining these data allows to determine which subsets of streamlines within a white matter bundle connect to specific functional regions in each individual. That is, this approach identifies the functionally defined white matter sub-bundles of the brain. We argue that this approach not only enhances the accuracy of interpreting the functional relevancy of white matter bundles, but also enables segmentation of these large-scale bundles into meaningful functional units, which can then be linked to behavior with enhanced precision. Importantly, this approach has the potential for making new discoveries of the fine-grained functional relevancy of white matter connections in the visual system and the brain more broadly, akin to the flurry of research that has identified functional regions in cortex.
\end{abstract}

Keywords White matter $\cdot$ Fascicles $\cdot$ Functional magnetic resonance imaging $\cdot$ Diffusion magnetic resonance imaging $\cdot$ Precision neuroscience

\section{White matter bundles of the human brain}

The white matter of the human brain contains a complex architecture of structural connections of different length and size that connect brain regions and enable them to communicate with each other. Large-scale connections that link distant parts of the brain are referred to as white matter fascicles. Most fascicles were initially identified in ex vivo

Mareike Grotheer

mareike.grotheer@uni-marburg.de

1 Department of Psychology, Philipps-Universität Marburg, 35032 Marburg, Germany

2 Center for Mind, Brain and Behavior-CMBB, Philipps-Universität Marburg and Justus-Liebig-Universität Giessen, 35037 Marburg, Germany

3 Psychology Department, Stanford University, Stanford, CA 94305, USA

4 Wu Tsai Neurosciences Institute, Stanford University, Stanford, CA 94305, USA dissection studies and later modeled from in vivo diffusion magnetic resonance imaging (dMRI) data, whereas anatomical priors from the dissection literature are used to guide dMRI analyses. dMRI tractography uses the diffusivity of water molecules within each voxel to estimate the trajectory of white matter connections, as water moves more freely along a connection than perpendicular to it (for a recent review, see Jeurissen et al. (2019)). Nonetheless, dMRI tractography is not without limitations (e.g. Bastiani et al. 2012; Jones et al. 2013; Thomas et al. 2014; Reveley et al. 2015; Maier-Hein et al. 2017) and can only provide a model of the fascicles. As such, here, we will use the term "bundle" when we refer to white matter fascicles estimated from dMRI data. Each bundle is made up of a large number of streamlines, i.e., individual lines traced in dMRI tractography. Importantly, we also use the term sub-bundle, which we define as a subset of streamlines within a bundle that form a meaningful functional and/or structural unit.

Fascicles of the brain are often divided into three main types: (1) projection fascicles, which connect between 
sub-cortical and cortical regions (e.g., the cortico-spinal tract (CST)), (2) commissural fascicles, which connect between the two hemispheres (e.g., forceps major (FMa) and forceps minor (FMi)), and (3) association fascicles, which connect distant cortical regions within a hemisphere. Well-studied association fascicles include: (1) the arcuate fasciculus (AF), which connects the frontal and temporal cortices (Catani et al. 2002), (2) the superior longitudinal fasciculus (SLF), which connects the frontal and parietal cortices (Catani et al. 2002), (3) the inferior fronto-occipital fasciculus (IFOF), which connects the frontal and occipital cortices (Catani et al. 2002), (4) the inferior longitudinal fasciculus (ILF), which connects the occipital cortex to the tip of the temporal lope (Catani et al. 2002), (5) the uncinate fasciculus (UCI), which connects the tip of the temporal lobe with the frontal cortex (Catani et al. 2002), (6) the vertical occipital fasciculus (VOF), which connects the ventral and dorsal occipital cortices (Yeatman et al. 2014; Takemura et al. 2016b; Weiner et al. 2016; Bullock et al. 2021) and (7) the posterior arcuate fasciculus (pAF), which connects the temporal and parietal cortices (Weiner et al. 2016) (Fig. 1a; for further details on these and other fascicles, see Bullock et al. 2021).

While fascicles by themselves do not have functional properties, they are thought to play an important role in brain function by connecting distant functional regions that are involved in a shared behavior (e.g., reading). Thus, understanding the functional role of fascicles has long-reaching implications for pre-surgical planning, identifying biomarkers of neurological and psychological disorders as well as for understanding brain development, organization, and plasticity, more generally. Ex vivo dissection studies have assigned functional relevancy to fascicles by linking behavioral deficits to lesions of particular fascicles (e.g. Catani and Ffytche 2005; Catani and Mesulam 2008). Additionally, dMRI studies have examined the relationship between behavioral metrics and diffusion metrics of bundles (e.g., the correlation between reading skill and fractional anisotropy in a bundle Yeatman et al. 2011; Vandermosten et al. 2012; Vanderauwera et al. 2017)) to link white matter fascicles to brain function.

In combination, dissection and dMRI approaches have provided valuable, yet somewhat simplistic insights on the functional relevancy of a given fascicle. After all, in both approaches, fascicles are considered as uniform structures that are linked to a specific brain function and/or behavior. The concern with this approach is that it assumes that the entirety of a large anatomical structure such as a fascicle, which is typically several $\mathrm{cm}$ wide and can be more than $10 \mathrm{~cm}$ in length, is involved in the relevant function. In

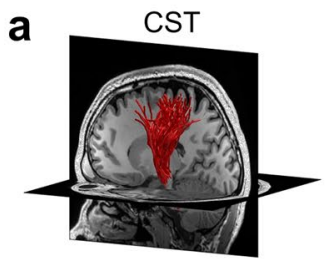

SLF

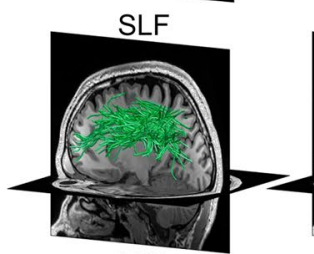

$\mathrm{UCl}$
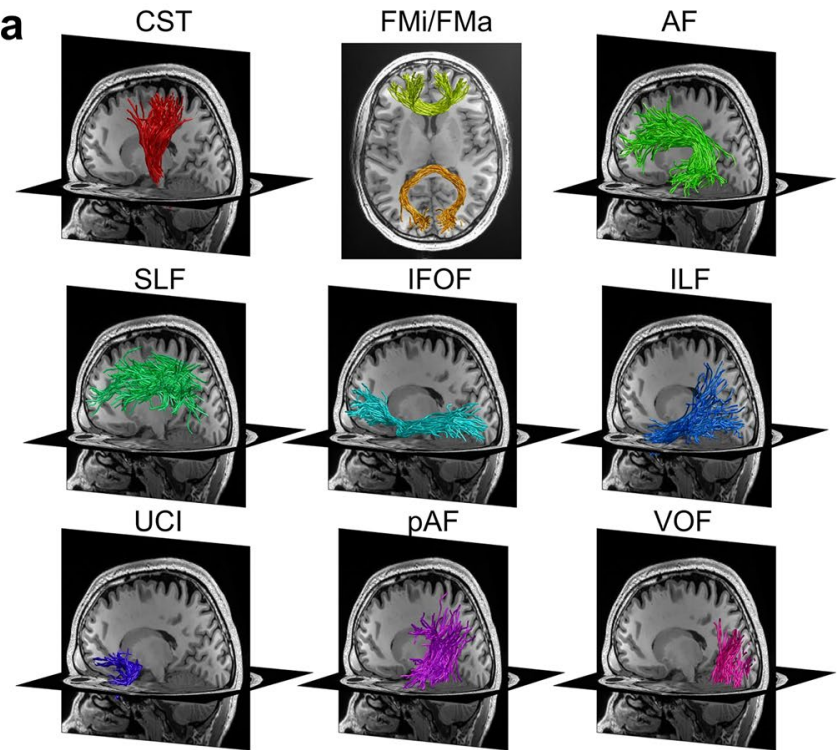

ILF

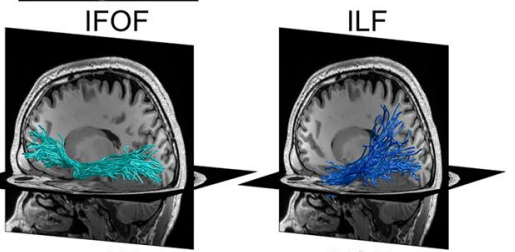

b

VOF
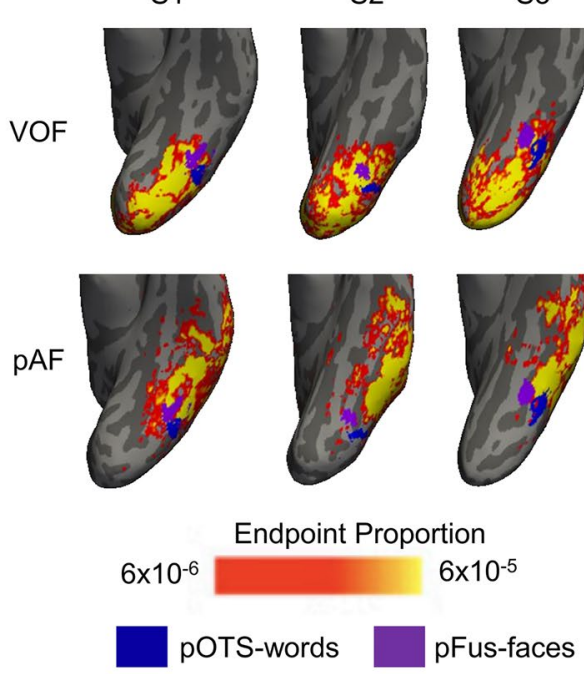
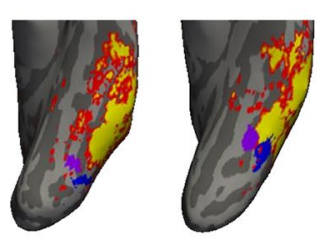

S3

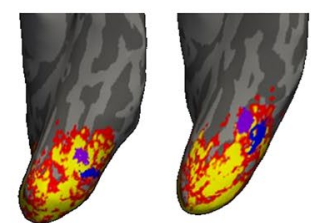

aces
Fig. 1 White matter bundles connect distant regions in the brain. a Examples of commonly investigated fascicles modeled with dMRI tractography. All panels shows bundles in the same representative individual. CST cortico-spinal tract, $F M a$ forceps major, $F M i$ forceps minor, $A F$ arcuate fasciculus, $p A F$ posterior arcuate fasciculus, $S L F$ superior longitudinal fasciculus, IFOF inferior fronto-occipital fasciculus, $I L F$ inferior longitudinal fasciculus, $U C I$ uncinate fasciculus, VOF vertical occipital fasciculus. b The proportion of VOF and pAF endpoints (yellow-reds) relative to category-selective regions (word- selective pOTS-words (blue), and face-selective pFus-faces (purple)) in the ventral temporal cortex. Probabilistic endpoint maps and fROIs are shown on the left inflated cortical surfaces of three example individuals. Endpoints of these bundles span a much larger spatial expanse than the fROIs and consequently connect to multiple functional regions. For example, the VOF endpoints connect to both faceand word-selective regions. $p O T S$ : posterior occipito-temporal sulcus, $p F u s$ : posterior fusiform gyrus, $f R O I$ : functional region of interest 
contrast, functional regions in cortex have a more granular organization. For example, the mosaic of category-selective regions in human ventral temporal cortex (Grill-Spector and Weiner 2014) includes several regions each about $\sim 1-2 \mathrm{~cm}$ in diameter. As such, the size of each category-selective region in ventral temporal cortex is at a finer spatial scale than the spatial extent of the endpoints of fascicles reaching ventral temporal cortex. This difference is spatial scales is illustrated in Fig. 1b, which shows, in 3 individuals, the relationship between cortical endpoints of the posterior arcuate fasciculus (pAF) and the vertical occipital fasciculus (VOF) in ventral temporal cortex and two visual category-selective regions: face-selective $\mathrm{pFus-faces} \mathrm{(also} \mathrm{called} \mathrm{fusiform} \mathrm{face}$ area 1) and word-selective pOTS-words (also called visual word form area 1). Critically, endpoints of both the pAF and VOF bundles span a larger spatial expanse than each of the functional regions, and the endpoints of the VOF extend across both face and word-selective regions. This difference in spatial scales suggests that these fascicles likely contribute to multiple functions (e.g., both face and word perception). Thus, we argue that, in order to understand the functional relevancy of fascicles in the brain, it is necessary to directly measure within individuals the set of regions to which each bundle connects.

\section{White matter bundles are heterogeneous in both structure and functional relevancy}

As discussed above, each white matter bundle likely connects regions that span multiple functional networks, suggesting that each bundle may contribute to multiple brain functions. Hence, when researchers analyze an entire bundle as a single entity, they will likely include streamlines that connect to regions from multiple networks. This, in turn, may make the derived measurements, such as the fractional anisotropy, less precise, because researchers may be averaging the measure of interest across streamlines that are involved in different functions and have different structural properties.

The idea that bundles are not uniform in their structural properties, and hence in metrics such as fractional anisotropy, is supported by recent work that has divided large bundles into sub-bundles. These sub-bundles can be identified based on structural criteria, in which case we will refer to them as structurally defined sub-bundles. Bundles may be structurally divided based on different spatial trajectories of groups of streamlines or based on structural differences between groups of streamlines within the bundle. For example, even though it is often considered to be a single bundle, the superior longitudinal fasciculus (SLF) has been divided into 2-3 distinct structural sub-bundles, inspired by findings in non-human primates (Petrides and Pandya 1984;
Schmahmann and Pandya 2006) (Fig. 2a). In humans, these structural sub-bundles have been identified based on differences in the spatial trajectories of streamlines (Makris et al. 2005; Thiebaut de Schotten et al. 2011; Wang et al. 2016) (Fig. 2b) or based on differences in the structural properties of streamlines (Schurr et al. 2020). While structurally defined sub-bundles still contain many streamlines, they are likely to be more homogenous than the entire bundle. Interestingly, analyses of the functional relevancy of the structural sub-bundles of the SLF suggest that they show different development trajectories and may be differentially impacted in different cognitive disorders (Galantucci et al. 2011; Thiebaut de Schotten et al. 2011; Parlatini et al. 2017; Fitzgerald et al. 2018; Amemiya et al. 2021). Other examples of structurally defined sub-bundles include: (1) the optic radiation, which contains an anterior sub-bundle that includes Meyer's loop, which has different structural properties than the rest of the bundle (Schurr et al. 2018), and (2) the anterior limb of the internal capsule, which in both non-human primates and humans contains distinct sub-bundles which vary in their connectivity to different parts of cortex (Safadi et al. 2018).

Sub-bundles can also be identified based on their connections to different functional regions in cortex, which we refer to as functionally defined sub-bundles (fSuB). For example, the SLF contains at least two different fSuB that run roughly in parallel: one sub-bundle consists of streamlines that connect distant cortical regions involved in reading, and the other consists of streamlines that connects regions involved in mathematical processing (Grotheer et al. 2019) (Fig. 2c). Interestingly, these fSuB of the SLF also differ in their structural properties; for example, R1 relaxation rate (which is linearly related to level of myelination Stüber et al. 2014; Kirilina et al. 2020)) is higher in the fSuB of the SLF involved in reading than the one involved in mathematical processing (Grotheer et al. 2019). Similarly, not only the SLF but also the arcuate fasciculus (AF) can be divided into two distinct fSuB (Fig. 2d), one involved in reading and the other in mathematical processing, which also vary in their $\mathrm{R} 1$ relaxation rate (Grotheer et al. 2019).

These examples highlight the tight interplay between structure and functional relevancy within individual white matter bundles, as well as the potential benefits of the increased spatial precision gained by dividing large-scale bundles into meaningful sub-bundles. Furthermore, these examples underscore that delineations of large-scale bundles into sub-bundles defined by distinct structural properties, is a promising data-driven approach to gain a finer grained understanding of the organization and functional relevancy of white matter connections (see also Schurr et al. 2018, 2019, 2020). Nonetheless, structurally defined sub-bundles may still connect to regions across multiple functional networks. Hence, we argue that if one's goal is to understand the functional relevancy of white matter connections, one 

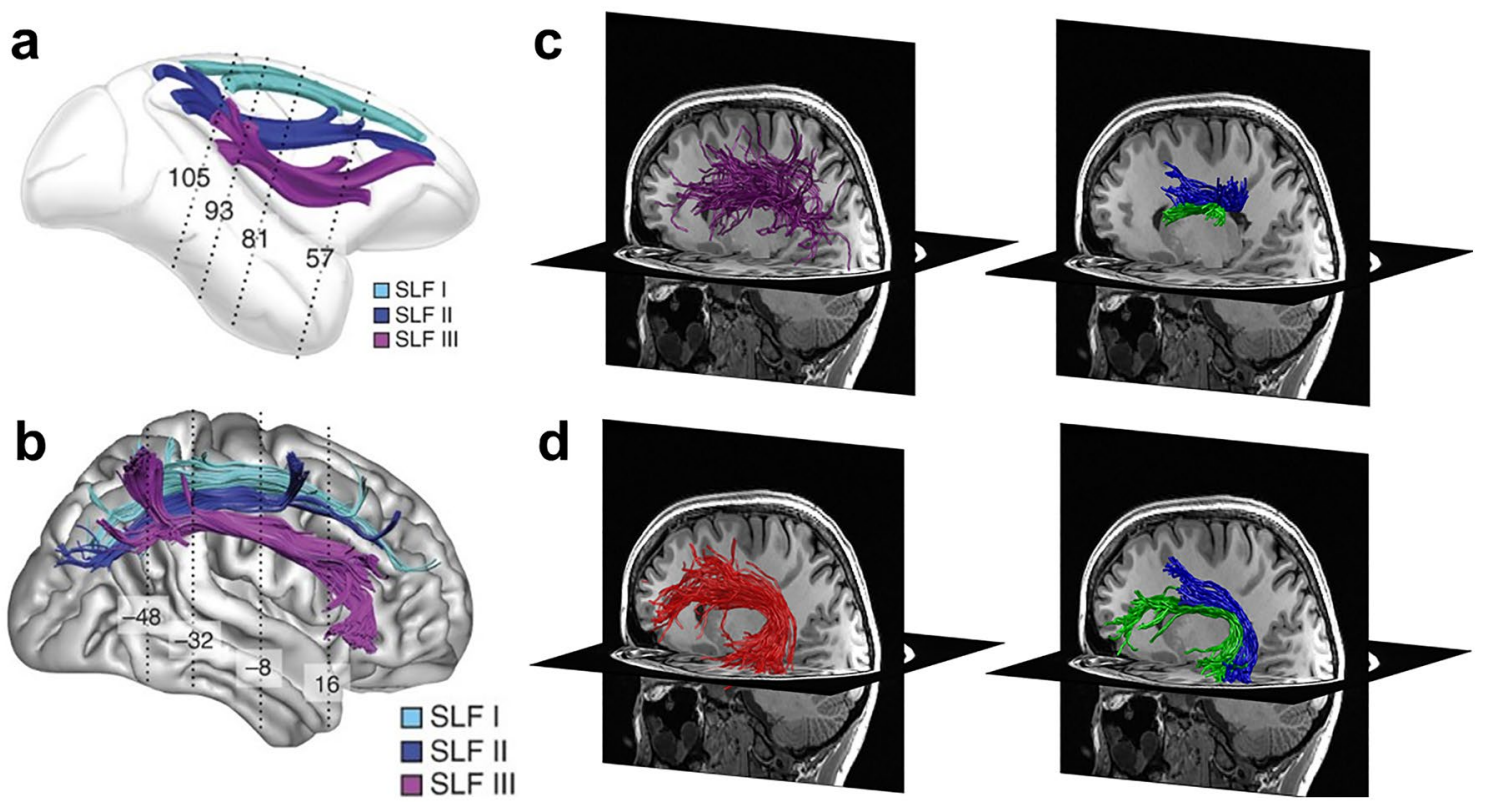

Fig. 2 The superior longitudinal fasciculus (SLF) and arcuate fasciculus $(\mathrm{AF})$ contain sub-bundles with distinct structural and functional properties. a, b Distinct structure: The SLF contains three distinct sub-bundles in monkeys (a) and in humans (b). Figure reproduced with modification from Thiebaut de Schotten et al. (2011). (c-d) Distinct function. c Comparison of the entire SLF (purple, left column) and sub-bundles within the SLF related to mathematical processing

should directly intersect functional regions and bundles within the same individual, that is directly identify the $\mathrm{fSuB}$ of a given functional network.

\section{Linking functional regions and white matter bundles in the visual system and beyond}

Over the past 25 years, researchers have used functional magnetic resonance imaging (fMRI) to identify regions of cortex involved in specific brain functions. Regions associated with a common function are thought to form brain networks, that together support important behaviors like recognizing faces or reading words. While white matter connections themselves are not thought to have functional properties, they nonetheless play an important role in brain function by connecting functional regions of the same network to one another. As such, identifying the structural connections between functional regions of a cortical network is important. After all, structural properties of these connections may impact the efficiency of communication within a brain network, and in turn its function (e.g. Thomas et al. 2009), and structural connections also constrain the location of functional regions (e.g. Saygin et al. 2016). (blue, right column) and reading (green, right column) within the same individual. d Comparison of the entire AF (red, left column) and sub-bundles within the AF related to mathematical processing (blue, right column) and reading (green, right column) within the same individual. c, d Reproduced with modifications from Grotheer et al. (2019). $A F$ arcuate fasciculus, $S L F$ superior longitudinal fasciculus

The human visual system is an excellent model system for examining the link between brain structure and function, as its functional organization is comparatively well understood and easy to probe. As such, during the last 10 years, much progress has been made in understanding the white matter connections of functional regions within the human visual system. This includes studies that explicated: (1) the intricate structural connections between functional regions of the face network (Gschwind et al. 2012; Pyles et al. 2013), (2) different white matter connections associated with face and place processing, respectively (Gomez et al. 2015), (3) the white matter connections of the visual word form area (Yeatman et al. 2013; Bouhali et al. 2014), (4) the white matter connections between early visual cortex and category-selective regions in ventral temporal cortex (Kim et al. 2006), and (5) the white matter connections between early visual cortex and retinotopic visual areas along the IPS (Greenberg et al. 2012).

While earlier studies on the link between functional regions and white matter connections in the visual system predominantly focused on local connections, here, we will focus on a recent expansion of this work that intersects functional regions identified in individual subjects with each individual's white matter bundles derived from that person's whole-brain tractogram. We argue that intersecting white matter bundles with functional regions in individual 
participant's brains is currently the most precise way to identify which streamlines within a bundle are connected to a specific functional network. It is critical that these analyses are done in individual participant's native brain space for three reasons (1) it enables higher precision in localizing functional regions of interest (fROIs, e.g. with a fMRI localizer), (2) it preserves relationships between function regions and particular macroanatomical structures like a sulcus or a gyrus, and (3) it preserves inter-individual differences in fROI and bundle size, shape, and location. This sensitivity to inter-individual differences is particularly important when examining special populations (e.g., children or patients) whose functional organization may differ from the general population and hence from available atlases based on brains of typical adults. Further, as this individual subject approach does not require spatial smoothing, which is commonly applied in group analyses but not in individual subject analyses, it further increases the spatial precision of the identified fROIs (e.g., Weiner and Grill-Spector 2013) and the subsequent identification of fSuB. Similarly, while there are stable functional-structural couplings in cortex, whereby functional regions are consistently located in specific sulci/ gyri (Van Essen et al. 2012; Weiner et al. 2017, 2018), defining the location of fROIs directly from a functional localizer is more accurate than predicting the region from cortical structure alone. Therefore, there is a limit to the degree to which anatomically defined regions can inform on the link between brain function and white matter connections.

Additionally, focusing on streamlines within bundles, rather than all streamlines in the tractogram, is advantageous as these large-scale bundles have been validated in ex vivo dissection studies. Thus, they are known to exist, which alleviates some of the common concerns regarding false positives in tractography (but see Maier-Hein et al. 2017). Moreover, focusing on these known anatomical structures also facilitates the interpretability of results and comparisons of results across studies. For these reasons, here, we review the promising approach of intersecting fROIs and white matter bundles within individual's native brain space to identify the functionally defined white matter sub-bundles of the brain.

\section{How to identify functionally defined white matter sub-bundles?}

To identify fSuB, researchers first use fMRI data to define functional regions of interest (fROI) in an individual's native brain space (Fig. 3a). For example, they may run a visual localizer (e.g., Kanwisher et al. 1997; Stigliani et al. 2015) to identify category-selective regions in the ventral temporal cortex, such as face-selective regions (fusiform face areas, also called mFus-faces and pFus-faces). Next, within the same individual's native brain space, a whole-brain white matter tractogram is created using dMRI data. The wholebrain tractogram is then classified into bundles (e.g., using automatic tools Yeatman et al. 2012; Garyfallidis et al. 2018; Grotheer et al., 2021a; Kruper et al. 2021). Next, the classified bundles are "intersected" with the fROI to isolate only those streamlines within each bundle that connect to that fROI, which generates the functionally defined white matter sub-bundles (fSuB) of that fROI. The generated fSuB are hence groups of streamlines from individual bundles that connect to a given functional region.

As outlined above, identifying $\mathrm{fSuB}$ requires the intersection of non-overlapping structures within individuals: fROIs that are located on the cortical surface, and white matter streamlines that lie under the cortical surface. As such, identifying $\mathrm{fSuB}$ has become feasible only in tandem with substantial improvements of tractography methods (such as constrained spherical deconvolution Tournier et al. 2012) and dMRI data quality (high angular resolution, multi-shell acquisitions, etc.). These methodological advances now enable tracing white matter streamlines all the way to the gray/white matter interface underneath the fROIs, increasing the precision of measurements. Indeed, earlier $\mathrm{fSuB}$ studies used spherical ROIs that were centered on fROIs but extended into the white matter to enable intersection with the white matter tractogram (Fig. 3b-top, e.g., Yeatman et al. 2013). As spherical ROIs are shaped differently and are often larger than the original fROIs, they reduce spatial precision. Better spatial precision than spherical ROIs can be achieved using another approach that combines dMRI data with anatomical segmentations of the individual's brain. In this approach, the subject's brain anatomy is segmented into gray and white matter and the gray/white matter interface is identified. Next, either the fROI is extended into the white matter or the streamline endpoints are extended into the gray matter, using the gray/white matter interface for guidance (e.g., Lerma-Usabiaga et al. 2018). Even higher precision can be achieved by leveraging anatomically constrained tractography (ACT, Smith et al. 2012). ACT utilizes each individual's brain segmentation and can seed tractography directly at the gray/white matter interface. This approach allows researchers to intersect streamlines and fROIs directly at the gray/white matter interface without extending either fROIs or streamlines, thereby further improving the precision of measurements (Fig. 3b, Grotheer et al. 2019). Together, these methodological innovations have enabled researchers to link between white matter connections and brain function with unprecedented precision.

When applying the functionally defined sub-bundles approach to study the functional relevancy of white matter connections, researchers should carefully consider several parameters that can impact the identified sub-bundles. These parameters include: the contrast and threshold used for fROI 


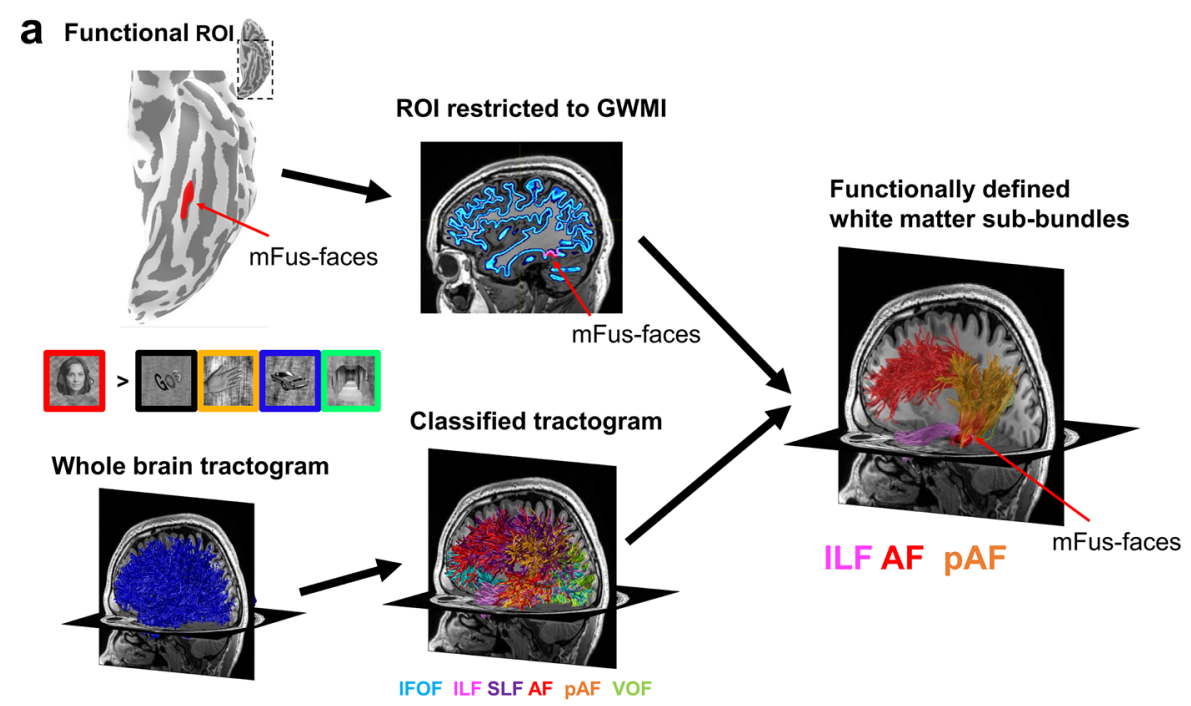

b Intersection with spherical ROI

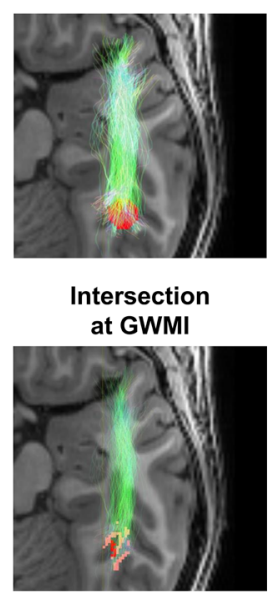

Fig. 3 By combining fMRI and dMRI, researchers can identify the functionally defined white matter sub-bundles (fSuB) that connect to a given functional region. a An overview of how to identify fSuB within a participant's brain. First, fMRI data is used to define a functional region of interest (fROI) in the cortex of an individual's brain. In this example, the fROI is mFus-faces, also referred to as fusiform face area 2 . The fROI can then be restricted to those voxels that fall at the gray/white matter interface. Next, dMRI data are used to generate a whole-brain tractogram in the same subject's native brain space, whereas seeding points for tractography can be placed uniformly across the gray/white matter interface. An example whole-brain tractogram is shown in blue. The tractogram is then classified into known bundles. Finally, by intersecting the fROI with the classified tracto-

definition, the quality of the dMRI data, and the tractography parameters, as detailed below.

First, when defining functional regions, it is recommended to use a stringent contrast that isolates the specific brain function one wishes to study from related functions. For example, when defining face-selective regions in the fusiform gyrus (pFus- and mFus-faces, also referred to as fusiform face areas), it is important to contrast brain responses to faces with those to many other categories (e.g., places, objects, words, and limbs) as this enables a more precise localization of these fROIs compared to when contrasting faces with only one other category (e.g., objects) (Weiner and Grill-Spector 2010). The statistical threshold used for fROI definition can then be chosen based on the literature to match previous measurements of the average size of the specific fROI. This is important, as larger fROIs will also lead to larger fSuB. Even when using the same contrast and threshold, the size of fROIs will vary between individuals, and it can hence be beneficial to run a control analysis where fROI size is kept constant across participants to ensure that betweensubject differences are not related to differences in their fROI sizes. gram, the $\mathrm{fSuB}$ of that fROI are identified. b Comparison of different approaches to intersecting fROIs and classified tractograms. The intersection can be done using spherical ROIs that are centered on the fROI and extend into the white matter (top) or directly at the gray/ white matter interface underneath the fROI (bottom). In this example, mFus-faces (red) is intersected with the inferior longitudinal fasciculus (green) in a representative subject. The fSuB generated at the gray/white matter interface is more precise than that identified using a spherical ROI. $f M R I$ functional magnetic resonance imaging, $d M R I$ diffusion weighted imaging, IFOF inferior frontal-occipital fasciculus, $I L F$ inferior longitudinal fasciculus, $S L F$ superior longitudinal fasciculus, $A F$ arcuate fasciculus, $p A F$ posterior arcuate fasciculus, $V O F$ vertical occipital fasciculus, GWMI gray/white matter interface

Second, when classifying tractograms into white matter bundles, researchers should be aware that each generated tractogram depends on the tracking algorithm (e.g., deterministic vs probabilistic) and other tracking parameters (Bastiani et al. 2012; Thomas et al. 2014; Takemura et al. 2016a; Maier-Hein et al. 2017). Furthermore, present tractography algorithms cannot fully resolve crossingfibers (Roebroeck et al. 2008; Maier-Hein et al. 2017) or accurately project into cortical gray matter (Reveley et al. 2015). To ensure that the identified $\mathrm{fSuB}$ reflect the underlying brain connections rather than parameter choices, we recommend generating multiple tractograms with different parameters and testing if and how results vary with the chosen parameters. Additionally, we recommend selecting an approach that identifies bundles which align well with anatomical priors from ex vivo dissection studies. Finally, it is advisable to stay up to date with the ongoing efforts to improve the accuracy of tractography methods (e.g. Smith et al. 2013; Pestilli et al. 2014; Takemura et al. 2016a). After all, as the tractograms become more accurate, so will the identified fSuB. 


\section{What have we learned from functionally defined white matter sub-bundles thus far?}

FSuB studies have advanced our understanding of the functional relevancy of the white matter bundles of the brain. One area of substantial recent progress is research on the white matter connections to a reading-related fROI, often referred to as the visual word form area (VWFA, Cohen et al. 2000; Dehaene and Cohen 2011). Importantly, $\mathrm{fSuB}$ studies using classified tractograms (Yeatman et al. 2013; Lerma-Usabiaga et al. 2018; Grotheer et al. 2019) revealed a consistent set of bundles (the arcuate fasciculus, vertical occipital fasciculus, inferior longitudinal fasciculus, and posterior arcuate fasciculus) that contain $\mathrm{fSuB}$ of the VWFA. These consistent findings across independent researchers, which used different methods to define fROIs, generate tractograms, and intersect the two, provides a striking example of the reliability and robustness of the $\mathrm{fSuB}$ approach. Moreover, these studies have generated important conceptual advances by: (1) showing that the VWFA contains two subregions that have distinct fSuB (the anterior VWFA (mOTS-words) connects predominantly to the posterior arcuate fasciculus and the posterior VWFA (pOTS-words) to the vertical occipital fasciculus (Lerma-Usabiaga et al. 2018)), (2) identifying the bundles (the arcuate fasciculus and posterior arcuate fasciculus) that connect the VWFA to other regions of the reading network (Grotheer et al. 2019), and (3) showing that fSuB related to reading and mathematical processing are spatially and structurally segregated within the arcuate fasciculus and the superior longitudinal fasciculus (Grotheer et al. 2019; Fig. 2c,d). Examination of fSuB have also been successfully implemented in non-human primates. For example, in the macaque monkey, this approach has been used to map the fSuB of the attention network (Sani et al. 2019).

Another exciting approach related to $\mathrm{fSuB}$ is using white matter bundles within individuals to explicate a functional region's white matter fingerprint, by grounding it to known white matter structures. The white matter fingerprint approach (Saygin et al. 2012, 2016; Osher et al. 2016) uses the white matter connectivity profile to predict functional maps in cortex. In brief, this method utilizes brain parcellations (e.g., anatomical parcels from FreeSurfer) and measures the amount of pairwise white matter streamlines between each voxel in one parcel to all other parcels in the brain, which is referred to as the white matter fingerprint (Saygin et al. 2012). Then, it finds a linear mapping (weighted sum) between the white matter fingerprint and a functional map of interest. This linear mapping can be used to predict functional maps in any new brain from its white matter fingerprint alone (Saygin et al.
2012, 2016; Osher et al. 2016). While the predictive power of this method is striking, its interpretability is limited, as it does not reveal which white matter bundles constitute the fingerprint that predicts a specific functional map. Thus, an exciting direction is combining the fingerprint approach with the $\mathrm{fSuB}$ approach to inform which bundles contribute to the prediction of functional responses. For example, in a recent paper, we combined these approaches and found that a weighted sum of the endpoint densities of the arcuate fasciculus, inferior longitudinal fasciculus and vertical occipital fasciculus successfully predicts distributed fMRI responses in ventral temporal cortex during a reading task, as well as the location of mOTS words (Grotheer et al. 2021b).

Another interesting extension of the fSuB approach is to move from intersecting white matter bundles with functional regions to intersecting them with functional maps. Several studies have combined measurements of retinotopic maps with tractography, providing insights on the retinotopic organization of the white matter (Dougherty et al. 2005; Yoshimine et al. 2018; Kurzawski et al. 2020; Movahedian Attar et al. 2020; Finzi et al. 2021). These studies (1) elucidated the retinotopic organization of streamlines crossing the splenium (Dougherty et al. 2005), (2) mapped short association fibers (u-fibers) in early visual cortex (Movahedian Attar et al. 2020), (3) investigated the effect of macular degeneration on the optic radiation (Yoshimine et al. 2018), (4) evaluated white matter connections between area prostriata and the thalamus, and (5) mapped the connections between face- and place-selective regions and eccentricity maps in early visual cortex (Finzi et al. 2021). While most of these studies focused on local connections rather than bundles, the $\mathrm{fSuB}$ approach can easily be adapted to such intersections with continuous functional maps in cortex. This, in turn, may provide an even finer spatial granularity.

Overall, the fSuB approach showed that: (1) the connections of a given functional region to the rest of the brain is typically supported by many white matter bundles rather than a single bundle, (2) a single white matter bundle connects to many functional regions that can be part of distinct functional networks, and (3) combining functional and diffusion MRI data within individuals allows the identification of sub-bundles specific to the brain function of interest, which not only improves precision but also provides a better understanding of the functional relevancy of the brain's white matter. Despite the progress made to date, the $\mathrm{fSuB}$ approach is still in its infancy and there are many additional ways in which it can advance understanding of the functional relevancy of white matter connections. For instance, the improved precision of $\mathrm{fSuB}$ may reveal novel biomarkers of neurological and psychiatric disorders that were hidden previously by averaging white matter properties across streamlines that support unrelated brain functions. Additionally, 
by identifying separate streamlines that are linked to different functions, the fSuB approach may be used to examine how learning and activity-dependent myelination impacts the white matter. Finally, fSuB can be used to study the link between functional development and the development of the white matter. For instance, it can be used to determine if structural differences between $\mathrm{fSuB}$ associated with different fROIs are present from birth, or if they develop during the lifetime of an individual.

\section{Conclusion}

We propose that the $\mathrm{fSuB}$ approach has the potential to significantly advance our understanding of functional-structural coupling in the visual system and the brain more broadly. As dMRI data quality and tractography approaches improve, so will the anatomical accuracy of the identified sub-bundles, which in turn, will enable researchers to focus on specific streamlines that connect functional regions within the brain network they are investigating. This increased precision will advance understanding of the link between diffusion metrics and behavior in typical as well as clinical populations. The increased precision afforded by the fSuB approach may further facilitate the integration of structural and functional connectivity measures, the detection of biomarkers for different neurological disorders as well as understanding of brain development, organization, and plasticity.

Acknowledgements This work was supported by the Center for Mind, Brain and Behavior (CMBB, Philipps-Universität Marburg and JustusLiebig-Universität Giessen) awarded to MG, NSF (DGE-1656518) awarded to EK and NIH (R01 EY023915) awarded to KGS.

Funding Open Access funding enabled and organized by Projekt DEAL.

\section{Declarations}

Conflict of interest Not applicable.

Availability of data and materials Not applicable.

Code availability Not applicable.

Open Access This article is licensed under a Creative Commons Attribution 4.0 International License, which permits use, sharing, adaptation, distribution and reproduction in any medium or format, as long as you give appropriate credit to the original author(s) and the source, provide a link to the Creative Commons licence, and indicate if changes were made. The images or other third party material in this article are included in the article's Creative Commons licence, unless indicated otherwise in a credit line to the material. If material is not included in the article's Creative Commons licence and your intended use is not permitted by statutory regulation or exceeds the permitted use, you will need to obtain permission directly from the copyright holder. To view a copy of this licence, visit http://creativecommons.org/licenses/by/4.0/.

\section{References}

Amemiya K, Naito E, Takemura H (2021) Age dependency and lateralization in the three branches of the human superior longitudinal fasciculus. Cortex 139:116-133. https://doi.org/10.1101/2021.01. 27.428023

Bastiani M, Shah NJ, Goebel R, Roebroeck A (2012) Human cortical connectome reconstruction from diffusion weighted MRI: the effect of tractography algorithm. Neuroimage 62:1732-1749

Bouhali F, Thiebaut de Schotten M, Pinel P, Poupon C, Mangin J-F, Dehaene S, Cohen L (2014) Anatomical connections of the visual word form area. J Neurosci 34:15402-15414. https://doi. org/10.1523/JNEUROSCI.4918-13.2014

Bullock DN, Hayday EA, Grier MD, Tang W, Pestilli F, Heilbronner S (2021) A taxonomy of the brain's white matter: twenty-one major tracts for the twenty-first century. PsyArXiv.

Catani M, Ffytche DH (2005) The rises and falls of disconnection syndromes. Brain 128:2224-2239

Catani M, Mesulam M (2008) The arcuate fasciculus and the disconnection theme in language and aphasia: history and current state. Cortex 44:953-961

Catani M, Howard RJ, Pajevic S, Jones DK (2002) Virtual in vivo interactive dissection of white matter fasciculi in the human brain. Neuroimage 17:77-94

Cohen L, Dehaene S, Naccache L, Lehéricy S, Dehaene-Lambertz G, Hénaff M-A, Michel F (2000) The visual word form area. Brain 123:291-307. https://doi.org/10.1093/brain/123.2.291

Dehaene S, Cohen L (2011) The unique role of the visual word form area in reading. Trends Cogn Sci 15:254-262

Dougherty RF, Ben-Shachar M, Bammer R, Brewer AA, Wandell BA (2005) Functional organization of human occipital-callosal fiber tracts. Proc Natl Acad Sci 102:7350-7355

Finzi D, Gomez J, Nordt M, Rezai AA, Poltoratski S, Grill-Spector K (2021) Differential spatial computations in ventral and lateral face-selective regions are scaffolded by structural connections. Nat Commun 12:2278

Fitzgerald J, Leemans A, Kehoe E, O'Hanlon E, Gallagher L, McGrath J (2018) Abnormal fronto-parietal white matter organisation in the superior longitudinal fasciculus branches in autism spectrum disorders. Eur J Neurosci 47:652-661

Galantucci S, Tartaglia MC, Wilson SM, Henry ML, Filippi M, Agosta F, Dronkers NF, Henry RG, Ogar JM, Miller BL, GornoTempini ML (2011) White matter damage in primary progressive aphasias: A diffusion tensor tractography study. Brain 134:3011-3029

Garyfallidis E, Côté MA, Rheault F, Sidhu J, Hau J, Petit L, Fortin D, Cunanne S, Descoteaux M (2018) Recognition of white matter bundles using local and global streamline-based registration and clustering. Neuroimage 170:283-295

Gomez J, Pestilli F, Witthoft N, Golarai G, Liberman A, Poltoratski S, Yoon J, Grill-Spector K (2015) Functionally defined white matter reveals segregated pathways in human ventral temporal cortex associated with category-specific processing. Neuron 85:216-228

Greenberg AS, Verstynen T, Chiu Y-C, Yantis S, Schneider W, Behrmann M (2012) Visuotopic cortical connectivity underlying attention revealed with white-matter tractography. J Neurosci $32: 2773-2782$ 
Grill-Spector K, Weiner KS (2014) The functional architecture of the ventral temporal cortex and its role in categorization. Nat Rev Neurosci 15:536-548. https://doi.org/10.1038/nrn3747

Grotheer M, Zhen Z, Lerma-Usabiaga G, Grill-Spector K (2019) Separate lanes for adding and reading in the white matter highways of the human brain. Nat Commun 10:420216

Grotheer M, Rosenke M, Wu H, Kular H, Querdasi FR, Natu V, Yeatman JD, Grill-Spector K (2021a) White matter myelination during early infancy is explained by spatial gradients and myelin content at birth. bioRxiv:2021.03.29.437583

Grotheer M, Yeatman J, Grill-Spector K (2021b) White matter fascicles and cortical microstructure predict reading-related responses in human ventral temporal cortex. Neuroimage 227:117669

Gschwind M, Pourtois G, Schwartz S, Van De Ville D, Vuilleumier P (2012) White-matter connectivity between face-responsive regions in the human brain. Cereb Cortex 22:1564-1576

Jeurissen B, Descoteaux M, Mori S, Leemans A (2019) Diffusion MRI fiber tractography of the brain. NMR Biomed 32:3785

Jones DK, Knösche TR, Turner R (2013) White matter integrity, fiber count, and other fallacies: the do's and don'ts of diffusion MRI. Neuroimage 73:239-254

Kanwisher N, McDermott J, Chun MM (1997) The fusiform face area: a module in human extrastriate cortex specialized for face perception. J Neurosci 17:4302-4311

Kim M, Ducros M, Carlson T, Ronen I, He S, Ugurbil K, Kim DS (2006) Anatomical correlates of the functional organization in the human occipitotemporal cortex. Magn Reson Imaging 24:583-590

Kirilina E, Helbling S, Morawski M, Pine K, Reimann K, Jankuhn S, Dinse J, Deistung A, Reichenbach JR, Trampel R, Geyer S, Müller L, Jakubowski N, Arendt T, Bazin PL, Weiskopf N (2020) Superficial white matter imaging: Contrast mechanisms and whole-brain in vivo mapping. Sci Adv.

Kruper J, Yeatman JD, Richie-Halford A, Bloom D, Grotheer M, Caffarra S, Kiar G, Karipidis II, Roy E, Chandio BQ, Garyfallidis E, Rokem A (2021) Evaluating the Reliability of Human Brain White Matter Tractometry. Aperture Neuro 1:1-25

Kurzawski JW, Mikellidou K, Morrone MC, Pestilli F (2020) The visual white matter connecting human area prostriata and the thalamus is retinotopically organized. Brain Struct Funct 225:1839-1853

Lerma-Usabiaga G, Carreiras M, Paz-Alonso PM (2018) Converging evidence for functional and structural segregation within the left ventral occipitotemporal cortex in reading. Proc Natl Acad Sci USA 115:E9981-E9990

Maier-Hein KH et al (2017) The challenge of mapping the human connectome based on diffusion tractography. Nat Commun 8:1-13

Makris N, Kennedy DN, McInerney S, Sorensen AG, Wang R, Caviness VS, Pandya DN (2005) Segmentation of subcomponents within the superior longitudinal fascicle in humans: a quantitative, in vivo, DT-MRI study. Cereb Cortex 15:854-869

Movahedian Attar F, Kirilina E, Haenelt D, Pine KJ, Trampel R, Edwards LJ, Weiskopf N (2020) Mapping short association fibers in the early cortical visual processing stream using in vivo diffusion tractography. Cereb Cortex 30:4496-4514

Natu VS, Gomez J, Barnett M, Jeska B, Kirilina E, Jaeger C, Zhen Z, Cox S, Weiner KS, Weiskopf N, Grill-Spector K (2019) Apparent thinning of human visual cortex during childhood is associated with myelination. Proc Natl Acad Sci USA 116:20750-20759

Osher DE, Saxe RR, Koldewyn K, Gabrieli JDE, Kanwisher N, Saygin ZM (2016) Structural connectivity fingerprints predict cortical selectivity for multiple visual categories across cortex. Cereb Cortex 26:1668-1683

Parlatini V, Radua J, Dell'Acqua F, Leslie A, Simmons A, Murphy DG, Catani M, Thiebaut de Schotten M (2017) Functional segregation and integration within fronto-parietal networks. Neuroimage 146:367-375

Pestilli F, Yeatman JD, Rokem A, Kay KN, Wandell BA (2014) Evaluation and statistical inference for human connectomes. Nat Methods 11:1058-1063

Petrides M, Pandya DN (1984) Projections to the frontal cortex from the posterior parietal region in the rhesus monkey. J Comp Neurol 228:105-116

Pyles JA, Verstynen TD, Schneider W, Tarr MJ (2013) Explicating the face perception network with white matter connectivity ptito. PLOS ONE 8:61611

Reveley C, Seth AK, Pierpaoli C, Silva AC, Yu D, Saunders RC, Leopold DA, Ye FQ (2015) Superficial white matter fiber systems impede detection of long-range cortical connections in diffusion MR tractography. Proc Natl Acad Sci USA 112:E2820-E2828

Roebroeck A, Galuske R, Formisano E, Chiry O, Bratzke H, Ronen I, Kim D, Goebel R (2008) High-resolution diffusion tensor imaging and tractography of the human optic chiasm at $9.4 \mathrm{~T}$. Neuroimage 39:157-168

Safadi Z, Grisot G, Jbabdi S, Behrens TE, Heilbronner SR, McLaughlin NCR, Mandeville J, Versace A, Phillips ML, Lehman JF, Yendiki A, Haber SN (2018) Functional segmentation of the anterior limb of the internal capsule: linking white matter abnormalities to specific connections. J Neurosci 38:2106-2117

Sani I, McPherson BC, Stemmann H, Pestilli F, Freiwald WA (2019) Functionally defined white matter of the macaque monkey brain reveals a dorso-ventral attention network. Elife 8

Saygin ZM, Osher DE, Koldewyn K, Reynolds G, Gabrieli JDE, Saxe RR (2012) Anatomical connectivity patterns predict face selectivity in the fusiform gyrus. Nat Neurosci 15:321-327

Saygin ZM, Osher DE, Norton ES, Youssoufian DA, Beach SD, Feather J, Gaab N, Gabrieli JDE, Kanwisher N (2016) Connectivity precedes function in the development of the visual word form area. Nat Neurosci 19:1250-1255

Schmahmann JD, Pandya DN (2006) Fiber pathways of the brain. Oxford University Press

Schurr R, Duan Y, Norcia AM, Ogawa S, Yeatman JD, Mezer AA (2018) Tractography optimization using quantitative T1 mapping in the human optic radiation. Neuroimage 181:645-658

Schurr R, Filo S, Mezer AA (2019) Tractography delineation of the vertical occipital fasciculus using quantitative T1 mapping. Neuroimage 202:116121

Schurr R, Zelman A, Mezer AA (2020) Subdividing the superior longitudinal fasciculus using local quantitative MRI. Neuroimage 208:116439

Smith RE, Tournier JD, Calamante F, Connelly A (2012) Anatomically-constrained tractography: improved diffusion MRI streamlines tractography through effective use of anatomical information. Neuroimage 62:1924-1938

Smith RE, Tournier JD, Calamante F, Connelly A (2013) SIFT: spherical-deconvolution informed filtering of tractograms. Neuroimage 67:298-312

Stigliani A, Weiner KS, Grill-Spector K (2015) Temporal processing capacity in high-level visual cortex is domain specific. J Neurosci 35:12412-12424. https://doi.org/10.1523/JNEUROSCI.4822-14. 2015

Stüber C, Morawski M, Schäfer A, Labadie C, Wähnert M, Leuze C, Streicher M, Barapatre N, Reimann K, Geyer S, Spemann D, Turner R (2014) Myelin and iron concentration in the human brain: a quantitative study of MRI contrast. Neuroimage 93:95-106

Takemura H, Caiafa CF, Wandell BA, Pestilli F (2016a) Ensemble tractography Jbabdi S, ed. PLoS Comput Biol 12:e1004692. https:// doi.org/10.1371/journal.pcbi.1004692 
Takemura H, Rokem A, Winawer J, Yeatman JD, Wandell BA, Pestilli F (2016b) A major human white matter pathway between dorsal and ventral visual cortex. Cereb Cortex 26:2205-2214. https://doi. org/10.1093/cercor/bhv064

Thiebaut de Schotten M, Dell'Acqua F, Forkel S, Simmons A, Vergani F, Murphy DGM, Catani M (2011) A lateralized brain network for visuo-spatial attention. Nat Preced 1-1

Thomas C, Avidan G, Humphreys K, Jung KJ, Gao F, Behrmann M (2009) Reduced structural connectivity in ventral visual cortex in congenital prosopagnosia. Nat Neurosci 12:29-31

Thomas C, Ye FQ, Irfanoglu MO, Modi P, Saleem KS, Leopold DA, Pierpaoli C (2014) Anatomical accuracy of brain connections derived from diffusion MRI tractography is inherently limited. Proc Natl Acad Sci USA 111:16574-16579

Tournier JD, Calamante F, Connelly A (2012) MRtrix: diffusion tractography in crossing fiber regions. Int J Imaging Syst Technol 22:53-66. https://doi.org/10.1002/ima.22005

Van Essen DC, Glasser MF, Dierker DL, Harwell J, Coalson T (2012) Parcellations and hemispheric asymmetries of human cerebral cortex analyzed on surface-based atlases. Cereb Cortex 22:2241-2262

Vanderauwera J, Wouters J, Vandermosten M, Ghesquière P (2017) Early dynamics of white matter deficits in children developing dyslexia. Dev Cogn Neurosci 27:69-77

Vandermosten M, Boets B, Poelmans H, Sunaert S, Wouters J, Ghesquière $P$ (2012) A tractography study in dyslexia: Neuroanatomic correlates of orthographic, phonological and speech processing. Brain 135:935-948. https://doi.org/10.1093/brain/awr363

Wang X, Pathak S, Stefaneanu L, Yeh FC, Li S, Fernandez-Miranda JC (2016) Subcomponents and connectivity of the superior longitudinal fasciculus in the human brain. Brain Struct Funct 221:2075-2092

Weiner KS, Grill-Spector K (2010) Sparsely-distributed organization of face and limb activations in human ventral temporal cortex. Neuroimage 52:1559-1573

Weiner KS, Grill-Spector K (2013) Neural representations of faces and limbs neighbor in human high-level visual cortex: evidence for a new organization principle. Psychol Res 77:74-97
Weiner KS, Yeatman JD, Wandell BA (2017) The posterior arcuate fasciculus and the vertical occipital fasciculus. Cortex 97:274-276

Weiner KS, Barnett MA, Lorenz S, Caspers J, Stigliani A, Amunts K, Zilles K, Fischl B, Grill-Spector K (2017) The cytoarchitecture of domain-specific regions in human high-level visual cortex. Cereb Cortex 27:146-161

Weiner KS, Barnett MA, Witthoft N, Golarai G, Stigliani A, Kay KN, Gomez J, Natu VS, Amunts K, Zilles K, Grill-Spector K (2018) Defining the most probable location of the parahippocampal place area using cortex-based alignment and cross-validation. Neuroimage 170:373-384

Yeatman JD, Dougherty RF, Rykhlevskaia E, Sherbondy AJ, Deutsch GK, Wandell BA, Ben-Shachar M (2011) Anatomical properties of the arcuate fasciculus predict phonological and reading skills in children. J Cogn Neurosci 23:3304-3317. https://doi.org/10. 1162/jocn_a_00061

Yeatman JD, Dougherty RF, Myall NJ, Wandell BA, Feldman HM (2012) Tract profiles of white matter properties: automating fibertract quantification. PLoS ONE. https://doi.org/10.1371/journal. pone.0049790\&type $=$ printable

Yeatman JD, Rauschecker AM, Wandell BA (2013) Anatomy of the visual word form area: Adjacent cortical circuits and long-range white matter connections. Brain Lang 125:146-155

Yeatman JD, Weiner KS, Pestilli F, Rokem A, Mezer A, Wandell BA (2014) The vertical occipital fasciculus: A century of controversy resolved by in vivo measurements. Proc Natl Acad Sci 111:E5214-E5223. https://doi.org/10.1073/pnas.1418503111

Yoshimine S, Ogawa S, Horiguchi H, Terao M, Miyazaki A, Matsumoto K, Tsuneoka H, Nakano T, Masuda Y, Pestilli F (2018) Agerelated macular degeneration affects the optic radiation white matter projecting to locations of retinal damage. Brain Struct Funct 223:3889-3900

Publisher's Note Springer Nature remains neutral with regard to jurisdictional claims in published maps and institutional affiliations. 